\title{
A PI-ALGEBRA WHICH IS NOT PI WHEN AN INVERSE IS ADJOINED
}

\author{
ROBERT A. INDIK
}

\begin{abstract}
An example is produced of an algebra, embedded in $2 \times 2$ matrices over a field, which is not PI when an element's inverse is formally adjoined. This example is used to show that the generic $2 \times 2$ matrices, a domain, has the same property.
\end{abstract}

In commutative algebra the procedure of adjoining the inverse of an element in a ring has proven useful. Recently work has been done towards generalizing the concepts of commutative algebra and algebraic geometry to PI-algebras [1], [2], [6]. In this paper it is shown that the algebra which is obtained from a subring of a ring of $2 \times 2$ matrices over an extension of the base field, by formal adjunction of the inverse of an element is not PI. We do this by describing an irreducible infinite dimensional representation and applying Kaplansky's theorem [4].

For $A$ and $B$ algebras over a commutative ring $C$, we shall use the notation of the free product $A{ }^{*}{ }_{C} B$ as found in [5]. This $C$-algebra is the coproduct in the category of $C$-algebras.

For $R$ an algebra over a field $k$, and $x$ an element of $R$, let $x^{\prime}$ denote the residue class of $z$ in the algebra $R{ }^{*}{ }_{k} k[z] /(z x-1, x z-1)$. Define $R\left\{x^{\prime}\right\}$ to be the above algebra.

Let $k\{X, Y\}$ be the algebra obtained by adjoining two generic $2 \times 2$ matrices to a field $k$.

TheOREM 1. $k\{X, Y\}\left\{X^{\prime}\right\}$ is not a PI-algebra.

To prove this theorem we shall work with another algebra. We define $A$ to be the quotient of the free algebra $k\{x, y\}$ by the relations $y^{2}=0$ and $y x^{i} y=0$, for $i>0$.

LEMMA 2. The set $S=\left\{x^{i} y x^{j}, x^{k} ; i, j, k \geqslant 0\right\}$ spans $A$ as a vector space over $k$.

Proof. The set $S \cup\{0\}$ is closed under multiplication, since $x^{i} y x^{j} x^{k} y x^{n}=$ 0 , for $i, j, k, n \geqslant 0$. Thus since $A$ is generated as an algebra by $x$ and $y$, the ring $A$ is spanned by $S$ over $k$.

Note. $S$ is actually a basis for $A$.

Received by the editors July 25, 1978 and, in revised form, October 18, 1978.

AMS (MOS) subject classifications (1970). Primary 16A08; Secondary 16A38.

Key words and phrases. Polynomial identity algebra, noncommutative localization. 
Proposition 3. $A$ can be embedded in the matrix algebra $M_{2}(k(u, v))$.

Proof. We define a map $\varphi$ from $A$ into $M_{2}(k(u, v))$ by

$$
\varphi(y)=\left|\begin{array}{ll}
0 & 1 \\
0 & 0
\end{array}\right|, \quad \text { and } \varphi(x)=\left|\begin{array}{ll}
u & 0 \\
0 & v
\end{array}\right| \text {. }
$$

This map is well defined since $\varphi(y)^{2}=0$ and $\varphi(y) \varphi(x)^{i} \varphi(y)=0$ for $i$ positive. The values of $\varphi$ on $S$ are as follows:

$$
\varphi\left(x^{k}\right)=\left|\begin{array}{cc}
u^{k} & 0 \\
0 & v^{k}
\end{array}\right| \text { for } k \geqslant 0
$$

and

$$
\varphi\left(x^{i} y x^{j}\right)=\left|\begin{array}{cc}
0 & u^{i} v^{j} \\
0 & 0
\end{array}\right| \text { for } i, j \geqslant 0
$$

Let $\alpha$ be an element of $A$. Since $S$ spans $A$ we may write $\alpha$ as $\sum_{i, j>0} a_{i j} x^{i} y x^{j}$ $+\Sigma_{k>0} b_{k} x^{k}$. Suppose $\varphi(\alpha)=0$. Then

$$
\varphi(\alpha)=\left|\begin{array}{cc}
\sum_{k>0} b_{k} u^{k} & \sum_{i, j \geqslant 0} a_{i j} u^{i} v^{j} \\
0 & \sum_{k>0} b_{k} v^{k}
\end{array}\right|=0
$$

in $M_{2}(k(u, v))$. Thus $b_{\mathbf{k}}=a_{i j}=0$ for $i, j, k \geqslant 0$. We may conclude that $\varphi$ is an embedding.

COROllary 4. A satisfies the polynomial identities of the $2 \times 2$ matrices.

Proposition 5. The algebra $A\left\{x^{\prime}\right\}$ is not a polynomial identity algebra.

Proof. Kaplansky's theorem [4] shows that it suffices to find an infinite dimensional irreducible representation with commuting ring $k$. Let $V$ be the space of finite Laurent polynomials:

$$
\left\{\sum_{n \in \mathbf{Z}} a_{n} z^{n}, a_{n} \in k \text {, all but finitely many } a_{n}=0\right\} .
$$

Define a representation of $A\left\{x^{\prime}\right\}$ on $V$ by:

$$
\begin{aligned}
x \cdot\left(\sum a_{n} z^{n}\right) & =\sum a_{n} z^{n+1}, \\
x^{\prime} \cdot\left(\sum a_{n} z^{n}\right) & =\sum a_{n} z^{n-1}, \\
y \cdot\left(\sum a_{n} z^{n}\right) & =a_{0} z .
\end{aligned}
$$

Since $x x^{\prime}=x^{\prime} x=1$, we will denote $x^{\prime k}$ by $x^{-k}$. The representation is well defined since for all $v$ in $V$,

$$
\begin{aligned}
x \cdot\left(x^{\prime} \cdot v\right) & =x^{\prime} \cdot(x \cdot v)=v, \\
y \cdot(y \cdot v) & =0
\end{aligned}
$$

and

$$
y \cdot\left(x^{i} \cdot(y \cdot v)\right)=0 \text { for } i \text { positive. }
$$

We shall now prove that this representation is irreducible. Given any nonzero $v$ in $V$, we may write $v$ as $\sum a_{n} z^{n}$, with $a_{j} \neq 0$ for some $j$. For any other vector $w$ in $V$ there is some integer $N>0$ so that $w=\Sigma_{-N}^{+N} b_{n} z^{n}$. We 
may write

$$
w=\sum_{-N}^{+N} b_{n} z^{n}=\sum_{-N}^{+N} b_{n} x^{n-1} \frac{1}{a_{j}} y x^{-j} \cdot v .
$$

Thus $A\left\{x^{\prime}\right\} \cdot v=V$ for all nonzero $v$ in $V$, and the representation is irreducible. Suppose $\beta$ is an endomorphism of $V$ commuting with the action of $A\left\{x^{\prime}\right\}$. Define $s_{i j}$ in $k$, for $i$ and $j$ integers by

$$
\beta\left(z^{i}\right)=\sum_{j} s_{i j} z^{j}
$$

Comparing $x^{k} \cdot \beta\left(z^{i}\right)$ and $\beta\left(x^{k} \cdot z^{i}\right)$ yields that $s_{i j}=s_{i+k j+k}$. Similarly the relation $y \cdot \beta\left(z^{i}\right)=\beta\left(y \cdot z^{i}\right)$ implies that $s_{i 0}=0$ for $i \neq 0$. Thus $s_{i j}=0$ for $i \neq j$, and $s_{i i}=s_{00}$. By linearity $\beta(v)=s_{00} v$ for all $v$ in $V$. Since $V$ is an infinite-dimensional irreducible $A\left\{x^{\prime}\right\}$ module with commuting ring $k$, Kaplansky's theorem yields that $A\left\{x^{\prime}\right\}$ is not a PI-algebra.

Since $A$ is embeddable in the $2 \times 2$ matrices, there is a map given by $X \mapsto x$ and $Y \mapsto y$ making $A$ the homomorphic image of $K\{X, Y\}$. This map can be extended so that $A\left\{x^{\prime}\right\}$ is a homomorphic image of $k\{X, Y\}\left\{X^{\prime}\right\}$. Thus $k\{X, Y\}\left\{X^{\prime}\right\}$ has the same irreducible infinite dimensional representation, and thus is not PI.

It is known [5, p. 63] that the generic matrix algebras are domains. Thus an example of a domain, satisfying the identities of the $2 \times 2$ matrices, which is not PI when an elements inverse is adjoined has been produced.

The observation that Proposition 5 implies Theorem 1 is due to M. Artin.

\section{REFERENCES}

1. M. Artin, On Azumaya's algebras and finite dimensional representations of rings, J. Algebra 11 (1969), 532-563.

2. Specialization of representations of rings (1977), preprint.

3. R. Indik, On inversion of elements in PI-algebras, Undergraduate thesis, MIT, 1978.

4. Irving Kaplansky, Rings with a polynomial identity, Bull. Amer. Math. Soc. 54 (1948), 575-580.

5. Claudio Procesi, Rings with polynomial identities, Dekker, New York, 1973.

6. William Schelter, Integral extensions of rings satisfying a polynomial identity, J. Algebra 40 (1976), 245-257.

Department of Mathematics, Princeton University, Princeton, New Jersey 08544 